\title{
Novel Technological Aspects of Radiation Therapy in Head and Neck Cancer
}

\author{
(1) Branislav JEREMIĆ,' (D) Ivane KILADZE, ${ }^{2}$ (D) Gökhan ÖZYiĞiT, ${ }^{3}$ (D) Nenad FILIPOVIĆ, ${ }^{4}$ \\ (1) Pavol DUBINSKY 5
}

\author{
'University of Kragujevac Faculty of Medicine, Kragujevac-Serbia \\ ${ }^{2}$ Department of Medical Oncology, Caucasus Medical Centre, Tbilisi-Georgia \\ ${ }^{3}$ Department of Radiation Oncology, Hacettepe University, Faculty of Medicine, Ankara-Turkey \\ ${ }^{4}$ BiolRC, Center for Biomedical Engineering, Kragujevac-Serbia \\ ${ }^{5}$ Department of Radiation Oncology, East Slovakia Institute of Oncology, Kosice-Slovakia
}

\section{Introduction}

Radiation therapy (RT) plays an important role in the overall armamentarium of treatment possibilities in head and neck cancer (HNC). Irrespective of stage and histology or primary tumor Subsite, it can be used for both cure and palliation and used as sole treatment modality or in combination with surgery and/or

\begin{abstract}
SUMMARY
Radiation therapy (RT) is an important treatment modality in head and neck cancer (HNC) irrespective of stage, histology, and location of the primary tumor in both curative and palliative setting, with or without other treatment modalities such as surgery or chemotherapy. Based on advances with better imaging and introduction of sophisticated software for treatment and planning systems, radiation oncology of HNC witnessed major advantages resulting in both improved local control and better sparing of organs at risk. From computed tomography to magnetic resonance imaging and introduction of positron emission tomography with various radiotracers it became possible not only to diagnose and stage HNC with more confidence but also to introduce these technologies in RT treatment planning, and to use it during the RT course for the evaluation of response and additionally sculpture RT fields. Furthermore, it became possible to predict outcome based on anatomic and metabolic changes in HNC. Community of radiation oncologists successfully adopted transition from two-dimensional to three-dimensional RT and then to intensity modulated RT, as well as stereotactic radiotherapy (either single- or multi-fraction) regimens. There is renewed interest in heavy particles with both neutrons, carbon-ions and protons, the latter two being used more frequently in the recent years. This review article summarizes the most important accepts of novel RT technologies in HNC.
\end{abstract}

Keywords: Head and neck cancer; radiotherapy, treatment.

Copyright $\odot$ 2021, Turkish Society for Radiation Oncology 


\section{Positron Emission Tomography (PET) with Com- puted Tomography (CT) and Magnetic Resonance Imaging (MRI)}

Besides its use in the diagnosis and staging, PET-CT has increasingly been used in both treatment planning and monitoring the treatment response of HNC, mostly with ${ }^{18}$ F-Fluorodeoxyglucosae (FDG). A number of non- ${ }^{18}$ F-FDG radiotracers also attracted significant attention in the past decade. Among hypoxia radiotracers, uptake changes of ${ }^{18} \mathrm{~F}$-Fluoromisonidazole (MISO) early during the $\mathrm{RT}+\mathrm{CHT}$ course was shown as useful tool in predicting treatment response.[3] It was also proposed it could guide clinical hypoxia-based RT planning, [4] including RT boosting based on PET definition of hypoxic volumes. [5] In one study,[6] with patients with HPV+ oropharyngeal carcinomas (OPCs) it enabled lymph node RT dose reduction which led to impressive 2-year locoregional control (LRC), DM free rate, and 2 -year OS of $100 \%, 97 \%$, and $100 \%$, respectively, with less toxicity. ${ }^{18} \mathrm{~F}$-Fluoroazomycinarabinofuranozide (FAZA) is another radiotracer exploring hypoxia and was shown to be capable of estimating the reduction of the hypoxic volume of patients scanned during the RT course.[7] However, the main challenge with hypoxic tracers was that hypoxic regions within the tumor regions are not static. Since hypoxic regions move continuously during radiotherapy course, the practical use of those tracers is questioned.

Copper-labeled radiotracers were used to predict response in patients undergoing a baseline PET scan before treatment[8] as well as in predicting response to neoadjuvant RT-CHT.[9] Amino acid methionine (MET) had also been investigated as L-[methyl- $\left.{ }^{11} \mathrm{C}\right]$ MET in its possible role in offering better delineation of tumors in the process of RT planning.[10] Some studies showed that it can be useful predictive or prognostic tool in heavy ion RT.[11] Some studies indicated its usefulness in side effects monitoring, since a correlation between parotid gland salivary flow and the metabolic clearance of the parotid was noted with the regional salivary clearance decreasing with increasing of the regional radiation dose.[12] Furthermore, individual radiation dose response of parotid glands could be measured by 11C-MET PET in patients with salivary gland cancers.[13] Finally, $\left[{ }^{18} \mathrm{~F}\right]$ fluorothymidine (FLT), radiopharmaceutical that trace cell proliferation, was used to monitor early response to RT since FLT uptake can significantly decrease between consecutive scans performed during RT.[14] Not only a change in FLT uptake during RT or RT-CHT was shown to be strong predictor of long-term outcome[14] but also metabolic tumor volume and the total lesion proliferation could also differentiate responders from non-responders.[15]
Serial FLT (performed before RT-CHT and during it) was also useful in documenting changes in tumor proliferation volume, shown to be of predictive of PFS.[16]

In addition to PET-CT, we recently also witnessed the use of hybrid whole-body PET-MRI in an attempt to successfully merge molecular imaging of PET and the high spatial resolution and high tissue contrast information from MRI. It has been used only sporadically in $\mathrm{HNC}$ with somewhat conflicting results when staging and restaging with PET-MRI were compared to PET-CT in primary or recurrent HNC of various HN subsites.[17] It was also shown that PET-MRI guided tumor delineation during the RT planning process can provide more information than other imaging.[18] German researchers developed an accurate and robust multimodal deformable image registration strategy and integrated combined PET/MR data into RT treatment planning. [19] They had showed that biologically individualized RT based on combined PET/MRI in terms of dose painting was possible. The same researchers also focused on image quality of RT-customized PET/MRI in HNC patients using a dedicated hardware setup. [20] Simultaneous PET/MRI using RT positioning aids was clinically feasible while image quality obtained with a RT setup met planning requirements indicating its use for personalized RT planning.

\section{Intensity Modulated Radiation Therapy (IMRT) and Stereotactic Body Radiation Therapy (SBRT)}

In the past 30 years, three-dimensional (3D) RT enabled higher RT doses and better sparing of organs at risks (OARs), leading to improved LRC, and less side effects of RT in HNC. Superior form of this treatment is IMRT which employs multiple radiation beams, each being subdivided into a smaller radiation beamlets with varying individual beamlet intensities. HNC was one of the first and most successful stories of the use of IMRT due to large volumes needing RT, and close proximity of OARs such as parotid, eyes or brain stem successfully being spared with the IMRT.[21,22] Dosimetric/planning studies have mostly documented superiority of various IMRT techniques over $2 \mathrm{D}$ or $3 \mathrm{D}$ RT in both the conformity and dose distribution, [23] irrespective of the primary tumor site as well as sparing OARs. On the other side, LRC and OS as well as quality of life, patient-related symptoms, or saliva flow rate[24] have only infrequently been used as endpoints. When investigated, frequently there was no improvement in LC control[25,26] likely due to a similar PTV coverage. Rare studies noted improved cancer specific survival (CSS) [27] or LRC and relapse-free survival (RFS). This was observed for exclusive RT while in the post-oper- 
ative setting IMRT offered better LC.[28] Almost all of these studies showed significant sparing of OARs, in particular xerostomia.[26,27] However, when survival analysis was focused on in nasopharyngeal carcinoma (NPC), Zhang et al.[29] used meta-analytic (MA) approach (eight studies, 3570 patients) to document significantly superior OS and LC in IMRT group versus 2D/3D. Using MA, Marta et al.[25] analyzed five prospective randomized clinical trials (PRCTs) with 871 patients of which $82 \%$ were those with NPC, showing no difference in OS and LRC. However, there was a significant reduction of Grade 2-4 xerostomia in IMRTtreated patients $(\mathrm{p}<0.0001)$. Gupta et al.[30] analyzed seven PRCTs with 1155 patients. Five studies used xerostomia as an endpoint while one study each used OS or LRC as an endpoint. IMRT led to reduction of 36\% in risk reduction (RR) in Grade $>2$ acute xerostomia and reduction of $56 \%$ in Grade $>2$ late xerostomia. Due to a $24 \% \mathrm{RR}$ reduction of $\mathrm{LRC}$ and $30 \% \mathrm{RR}$ reduction in OS, authors called for a cautious interpretation of their results since the latter results were observed only in NPC patients and having analyzed only two studies.

Initially, the IMRT was used either as serial tomotherapy, step-and-shoot (SS) or dynamic/sliding window (SW) approach and was done sequential way, with its two phases built on experience obtained from the era of 2D/3D RT.[31] Past two decades witnessed major emphasis being placed on the use of arc approaches, most notably helical tomotherapy and intensity modulated arc therapy (IMAT) and the latter's subsequent and advanced form, known as volumetric modulated arc therapy (VMAT). VMAT was expected to bring advantage over IMRT or IMAT due to its enhanced flexibility in the delivery by facilitating alternating dose rate and gantry speed during dynamic movements of accelerator jaws and multileaf collimators, allowing the whole target to be treated using 1 or 2 arcs, although complex cases may require more.

A special advantage of IMRT is that it enables inhomogeneous dose distributions to be delivered to various volumes (primary and elective) with different dose per fraction without increasing the overall treatment time, the technique called simultaneous integrated boost (SIB). SIB allows all volumes to be treated within the single treatment plan without matching RT fields. With SIB technique clinicians started irradiating three clearly different (risk-wise) areas at the same time. It also enabled increase in the dose per fraction to the boost volume (e.g., $2.2 \mathrm{~Gy} /$ fraction), while, at the same time, kept the dose to the low risk/elective volume at a lower level (e.g., $1.6 \mathrm{~Gy} /$ fraction). SIB IMRT approach was shown to be dosimetrically better than sequential IMRT[31] and was more practical due to using a single plan from the start. Recent MA[32] compared sequential boost IMRT with SIB IMRT in HNC (seven studies and 1049 patients). Interestingly, there was no difference in any of the endpoints used; OS $(\mathrm{p}=0.71)$, PFS $(p=0.79)$, LRFS $(p=0.91)$, and DMFS $(p=0.63)$ including no difference in side effects. However, they contrasted previous findings that SIB was better than sequential IMRT,[33] leading to less side effects, [34] others showed superiority of sequential IMRT[35] due to a better coverage of the high dose regions, conformity and homogeneity, including less monitor units (MUs) being used.

Most recent planning studies compared several IMRT techniques showing similar PTV coverage, but improved homogeneity with 2 arcs with VMAT versus fixed field/SS IMRT.[36] While mean doses to the OARs were lower for VMAT with 2 arcs versus SW, VMAT also offered improved sparing of the contralateral parotid with a comparable PTV coverage compared to SW IMRT.[36] Double arc VMAT was superior to a single arc VMAT regarding PTV coverage and OAR sparing.[37] Contrasting these, the study of Bertelsen et al.[38] showed that a single arc VMAT may be either similar (PTV coverage) or only slightly better (elective nodal coverage) in patients with OPC or hypopharyngeal cancers. Other observed lower integral doses to the body with VMAT plans, [36] while other showed that with tomotherapy one can achieve better coverage of the low risk (elective) areas and can also achieve better dose conformity than VMAT or IMRT.[39] When doses to OARs have been evaluated, lowest dose for mandible was achieved with VMAT, all other organs with tomotherapy. One should not forget that with VMAT there is up to $50 \%$ reduction in $\mathrm{MU},[35,36]$ an important aspect in the daily work of the busy departments of radiation oncology worldwide. Not to be forgotten, too, is that in spite of shorter delivery time with VMAT, [35,39] it remains vitally dependent on the number of fields used in IMRT plans. In one study[40] in patients with OPC, rotational/arc IMRTs were preferable to SS/SW due to a faster fraction delivery and better sparing of OARs without a higher integral dose.

Stereotactic RT was also used in the primary treatment of HNC, mostly as a boost given after previous either IMRT or conventional RT. Single or fractionated stereotactic radiosurgery (SRS) or fractionated SBRT proved to be feasible and effective in the boost phase of the comprehensive RT treatment. [41,42] The Korean 
study[42] reported on 24 patients with extracranial HNC, mostly consisting of NPC $(n=19)$, treated with fractionated stereotactic RT as a boost. The median boost dose to NPC was 16 Gy (range, 8-40 Gy) after the median conventionally fractionated RT dose of 55.8 Gy (range, 36-61.2 Gy). Complete response was seen in $95 \%$ patients with LC rates and OS at 4 years being $89 \%$ and $75 \%$, respectively, achieved without occurrence of unexpectedly severe complications (one mucosal necrosis which eventually and completely healed). Subsequent reports in a small patient cohorts reconfirmed feasibility and efficacy of both single and multifraction SBRT. Siddiqui et al.[43] reported on ten primary HNC treated with single fraction of $13-18 \mathrm{~Gy}$ or $36-48 \mathrm{~Gy}$ in 5-8 fractions to obtain tumor control rate of $66.7 \%$ at 2 years with the median survival time (MST) of 28.7 months and 2-year OS of 50\%. Grade 3 side effects were seen only in two patients after 36 and 48 Gy given in 6 and 8 fractions, respectively. Several single institutional studies with limited number of patients used SBRT as a boost with 28 fractions delivering total doses ranging 10-38 Gy and reporting on MSTs of $>31.5$ months with a 3-5-years OS of 46.2-60\%.[44] Most recently, Baker et al.[45] provided detailed analysis and the long-term data on 195 patients with OPC treated with fractionated SBRT boost $(3 \times 5.5 \mathrm{~Gy})$ after IMRT was initially been given with $46 \mathrm{~Gy}$ in 23 daily fractions. Five-year OS, DSS, LC, and RC as well as late grade $>3$ toxicity were $67 \%, 85 \%, 90 \%, 93 \%$, and $28 \%$, respectively.

In a SRS domain, single fractions were used to boost NPC after initial RT was given with conventionally fractionated RT. Chang et al.[41] treated 23 patients with Linac-based technique delivering the median of $12 \mathrm{~Gy}$ (range 7-15 Gy) following the median of $66 \mathrm{~Gy}$ (range 64.8-70 Gy) of conventional RT. In all 23 patients (100\%) receiving SRS, following conventional RT-LC was achieved at a mean follow-up of 21 months (range 2-64 months) with no SRS-related complications. SRS delivered through Gamma Knife (GK) was also used as planned boost after RT-CHT in cases of selected sinonasal cancers and NPCs.[46] The mean initial RT dose delivered by IMRT was $64.3 \mathrm{~Gy}$ (range, 54$70 \mathrm{~Gy})$ at $2 \mathrm{~Gy}$ per fraction. After the median interval of 2.2 months from the end of IMRT, SRS boost with the median margin dose of 13 Gy (range, 12-20 Gy) was delivered. All patients achieved local control with no Grades 3-5 toxicity. Robotic SRS using the RT linear accelerator known as Cyber Knife was also used in either primary as SRS only $(n=6)$, or as a SRS boost $(n=7)$ or in post-operative setting $(\mathrm{n}=8)$ or for re-irradiation $(n=6)$ in the study of Ozyigit et al.[47] in 27 cases of nose and paranasal cancers. The median dose to the tumor was $31 \mathrm{~Gy}$ (range, 15-37.5 Gy) in median of 5 fractions (range, 3-5 fractions). LC was seen in $>75 \%$ cases with the 2-year survival for the whole group of $77.1 \%$ which was accompanied with $7 \%$ cases of brain necrosis and visual disorder each, bone necrosis in further $7 \%$ while $4 \%$ of patients experienced trismus.

Both IMRT and SBRT had also been used to treat recurrent disease. Majority of studies were single-institutional, retrospective reports on a small number of patients and unfortunately, with different patient, tumor and treatment (RT, surgery, and $\mathrm{CHT}$ ) characteristics making any firm conclusion rather impossible. Nevertheless, recent report[48] recently summarized the results in the setting of recurrent HNC. For the IMRT and SBRT, respectively, the median (and the range) of 2-year OS was 49\% (32-59\%) and 29\% (28-58\%), respectively. Corresponding figures for the LRC were $62 \%(52-67 \%)$ and $52 \%(28-64 \%)$, respectively. These results have been achieved with a variety of RT dose and fractionation characteristics. Ozyigit et al.[49] reported on a retrospective study comparing 3D RT (57 Gy in 2 Gy per fraction) versus SBRT (30 Gy over 5 consecutive days). No difference was found in LC rates or CSS rates, but serious late toxicities were more frequent in $3 \mathrm{D}$ RT group ( $48 \%$ vs. $21 \%$, p=0.04). Interestingly no difference was found in the fatal complications in the two groups of patients. Summarizing the existing literature, Alterio et al.[50] indicated that with standard fractionation, the dose of $>60$ Gy may be preferable, while in the case of SBRT, the dose equivalent to $40 \mathrm{~Gy}$ in 5 fractions seemed necessary, in both cases focusing on visible tumor. When reirradiation was used in the post-operative setting; however, it did not lead to significant improvement in OS. It offered better LRC and DFS, but at the expense of severe acute toxicity.[51] These side effects have also been significant burden in exclusive reirradiation series, including documented cases of carotid blowout syndrome (CBOS). As documented by Ho and Phan,[48] although not very frequent (1-8\%) CBOS is still fatal in most patients. While some experienced higher incidence of CBOS, [52] going as high as $17 \%$ with $15 \%$ dying of it, simple measures have been proposed (administering SBRT every other day, limiting median carotid artery dose to $<34 \mathrm{~Gy}$, excluding patients with a tumor surrounding $>180^{\circ}$ of the carotid artery) to minimize the risks.[53] Other late high grade $(>3)$ toxicity remains a much more frequent event, although one may notice somewhat lower rate with SBRT (7\%) when compared to IMRT (39\%). In addition to fractionated SBRT, Oda 
et al.[54] reported on GK SRS after previous fractionated RT in 14 patients of which 11 had NPC. Tumor margin doses ranged 10-27 Gy (median, 15 Gy), and the maximal tumor doses ranged 22-40 Gy (median, 28 Gy). Response rate (RR) was $43 \%$, while stable disease was in 14 of the patients. A second SRS was performed in four out of six re-growing tumors, of which response was seen in three, making the total control rate of $79 \%$.

Finally, important, although still sporadic, reports highlighted the advantage of IMRT over 3D regarding their respective cost-effectiveness. [55] They have included different health-care systems of different countries but unequivocally showed that IMRT was considered more cost effective than 3D. What these studies did not include were other benefits IMRT likely carries. These include shorter treatment times when VMAT is used, as well as lower short- and long-term costs related to toxicities (xerostomia, dysphagia, and dental problems), such as intensive supportive care which is frequently needed in HNC patients treated with intensive radical RT/CHT.[56]

\section{Heavy Particles Carbon Ions}

Carbon ions have also been used to treat both primary and recurrent non-squamous cell HNC. In the Japanese experience, 289 patients with adenoid cystic carcinoma (ACC) of the head and neck,[57] estimated 5 -year OS, PFS, and LC rates were $74 \%, 44 \%$, and $68 \%$, respectively. Of all patients, $15 \%$ experienced grade $\geq 3$ late toxicity, osteoradionecrosis (ORN) of the jaw bone being the most common. Two patients $(0.7 \%)$ treated for NPC died from a bleeding ulcer at the tumor site. In 26 patients with mucoepidermoid carcinoma,[58] the 3 -year rates of LC, PFs and OS were $95 \%, 73 \%$, and $89 \%$, respectively. Acute and late toxicity were judged to be moderate with no Grade 5 toxicities.

The German researchers[59] treated 229 patients with recurrent HNC of which $54.1 \%$ were ACC, $26.2 \%$ were squamous cell carcinomas, $8.3 \%$ were adenocarcinomas, and $11.4 \%$ were other tumor entities. The median local PFS was 24.2 months, and the median OS was 26.1 months. Acute grade $\geq 3$ toxicity was rare, while late toxicities were of grades $>3(n=18 ; 14.5 \%)$ only. When carbon ion RT was coupled with IMRT in high-risk NPC, [60] the estimated 5-year LC, DPFS, and OS rates were $90 \%, 86 \%$, and $86 \%$, respectively. There were $20 \%$ acute and $16 \%$ chronic Grade 3 side effects, respectively, and no toxicity $>3$ was observed. Adding carbon ion boost to IMRT was also used in 52 patients with ACC of the minor salivary gland tumors of the nasopharynx.[61] The estimated 5-year LC, DPFS, and OS were $49 \%, 54 \%$, and $69 \%$, respectively. Overall, Grade 3 toxicity was moderate with $12 \%$ acute and $8 \%$ late side effects. In a Phases I-II (ACCEPT) study,[62] Cetuximab was added to RT composed of IMRT and carbon ion boost to treat 23 patients with ACC of the $\mathrm{HN}$. Nine patients underwent surgery, none of which was R0. There was no Grades 4-5 toxicity. The 3-year DFS was $67 \%$, and median OS was 54 months. In a setting of a Phase II study,[63] patients with various malignant salivary gland tumors were treated with carbon ions followed by IMRT. Grade 3 mucositis was observed in $26 \%$ of patients and $38 \%$ patients reported adverse events of the ear. The most common observed late effects were Grade 1 xerostomia (49\%), hearing impairment (25\%), and adverse events of the eye (20\%), with no visual impairment or loss of vision. Grade 1 central nervous system necrosis occurred in $6 \%$, and 1 Grade 4 internal carotid artery hemorrhage without neurologic sequelae. Three-year the LC, PFS, and OS were $81.9 \%, 57.9 \%$, and $78.4 \%$, respectively.

\section{Neutrons}

Neutrons have been used primarily for salivary gland tumors and only rarely reports included non-squamous cell carcinomas. The LC rates for advanced salivary gland tumors were mostly around $60-75 \%$. $[64,65]$ Recently, Stannard et al.[66] reported on an experience where the median dose 20.4 Gy was given in 12 fractions in 4 weeks or in 15 fractions in 5 weeks to 335 patients which included 176 unresectable, 104 macroscopically residual, and 55 unresected tumors. LRC was $39.1 \%$ at 10 years and DSS was $53.7 \%$ at 10 years. In majority of published studies, Grades 3-4 late toxicity was around $10-15 \%$ at 5-10 years. Some studies, however, reported on higher incidence of toxicity, such as that of Maor et al.[67] who reported on $>$ Grade 3 late toxicity being observed in $39.7 \%$. In their study, Grade 4 ORN occurred in four patients (5.9\%). This treatment approach has largely been abandoned today and is only sporadically practiced in few centers worldwide.

\section{Protons}

With clinical data slowly emerging, dosimetric studies brought better understanding of both advantages and challenges with this treatment modality in HNC. Spot-scanned beams and intensity modulated proton beams (IMPT) were shown to provide better sparing of OARs when compared to scattered proton beams. [68] IMPT allowed extraordinary conformity of treatment plans and dose escalation in clinical scenarios 
when OARs such as optic chiasm and/or optic nerves in the immediate vicinity of paranasal sinus tumors. [69] Normal tissue control probability (NTCP) models confirmed the benefit of using IMPT in cases of NPC to decrease the dose to parotid glands,[70] to swallowing muscles[71] or to oral cavity and spinal cord.[72] Data pointed to ipsilateral and well lateralized targets in the neck as preferable for protons. On the other side, when more central and or/bulky or bilateral target volumes need to be treated, delivery of IMPT may be faced with significant uncertainty of delivered dose deposition due to both anatomic and physical properties of both the patient and the tumor.[73] Among efforts to address these issues and increase robustness of IMPT planning, multi-field optimization (MFO)[74] and weekly verification scans and adaptive re-planning[75] have been proposed. More recent studies reconfirmed the feasibility of improving tumor coverage and reducing integral dose to OARs with MFO-IMPT relative to IMRT and helical tomotherapy in cases of NPC.[76] In the postoperative setting of OPC, too, dosimetric superiority of IMPT over IMRT or VMAT was also suggested.[76]

Still the vast majority of reports and patients therein were of non-SQC histology. Several single-institutional series[77,78] reported on chordomas and chondrosarcomas as well as nasal cavity and paranasal sinus cancers, some of which, however reported on high rates of late toxicity (42\%) which may have compromised good LC (4-year, 54\%),[77] but with higher doses LC was achieved in $70-100 \%$ and for prolonged periods of time. [78] In the first long-term report of 64 patients with the base of skull tumors treated with protons, [79] 44 were treated with spot scanning and 20 with IMPT. High median total doses for chordomas and chondrosarcomas were given to achieve 5-year LC of $81 \%$ and $94 \%$ for the two histologies, respectively. The corresponding figures for OS were $100 \%$ and $91 \%$, respectively, accompanied with limited toxicity and no brain stem injury.

In NPC, with or without photons, [80] excellent LC (up to $100 \%$ ) and OS (28 months) were observed. Even in T4 tumors, local failure was around 6\% after 3.5 years. However, late toxicities (radiographic temporal lobe changes) were frequently observed (29\%). Recent reports on the use of IMPT, however, point toward the decrease in toxicity when compared to IMRT.[81] Gastrostomy tube dependency (20\% vs. $65 \%$ ) significantly favored IMPT as a consequence of improved oral cavity sparing as was confirmed in other studies, too.[82]

In the nose and sinonasal region, protons also proved to offer better dosimetry, and safe dose escalation which was coupled with reduced side effects and improved results (LC in 90\% cases) in various histological forms. [83] When protons have been compared to IMRT in patients with nasopharyngeal, nasal cavity and paranasal sinus cancer, protons offered improved sparing of oral cavity, esophagus, larynx, and parotid glands.[84] When prolonged follow-up was provided,[85] LC was $50 \%$ at 5 years, with $16 \%$ Grade 3, and $11 \%$ Grade 4 toxicity, but most commonly being of wound complications. For non-surgical candidates, too, passively scattered proton therapy provided good 2 - and 3-years OS rates of $>60 \%$ and LC rates of $70-95 \%$ observed with mixed histologies and disease stages.[86]

Rare reports provided the data about feasibility of using protons in periorbital tumors. In one such retrospective study,[87] 13 out of 14 operated patients with primary lacrimal sac or nasolacrimal duct carcinomas, received post-operative RT with protons or IMRT with a median dose of $60 \mathrm{~Gy}$, while eight patients received $\mathrm{CHT}$. With the globe spared in all $(\mathrm{n}=10)$ non-exenterated tumors, $90 \%$ of patients either maintained or improved visual acuity. Another report[88] on 20 patients with orbital and ocular adnexa tumors provided results after orbit-sparing surgery, followed by protons. After 60 Gy (RBE), there were no local recurrences after a median follow-up of 27 months, but there were one regional and one distant recurrence (total, 10\%). Treatment was well tolerated with only $20 \%$ of patients having a decrease in visual acuity.

OPC is a another cancer where improvement of results with IMPT is expected largely due to significant change toward more HPV+ patients in recent years. [2] When accelerated photon RT and concurrent proton boost were used in 29 patients with advanced OPC,[89] only 3 (11\%) late Grade 3 toxicity was observed with LC of $84 \%$ at 5 years. In the setting of OPC, MFO IMPT seems as mandatory for covering complex bilateral target volumes with successful delivery. In one such attempt, researchers used IMPT in 26 p16+ OPC to achieve low rates of Grade 3 mucositis (15\%) and $19 \%$ of patients required feeding tube, which compared favorably with the historical (IMRT) rates of 48\%.[90] In a case-matched analysis[91] with 50 IMPT and 100 IMRT, there was no difference in OS $(\mathrm{p}=0.44)$ or in PFS $(\mathrm{p}=0.96)$. When considering the pre-planned composite endpoint of Grade 3 weight loss or G-tube presence, the ORs were $\mathrm{OR}=0.44 ; \mathrm{p}=0.05$ at 3 months after treatment and $\mathrm{OR}=0.23 ; \mathrm{p}=0.01$ at 1 year after treatment. One study[92] reported on 50 patients treated with IMPT (92\%, MFO), of which $98 \%$ had Stage III/IV disease, $64 \%$ received concurrent therapy, and 35\% received induction CHT. Importantly, $98 \%$ were $\mathrm{p} 16$ pos- 
itive. No grade $>4$ toxicities were observed. The 2-year OS and PFS rates were $94.5 \%$ and $88.6 \%$, respectively.

Protons were also used in reirradiation of HNC patients with recurrent or progressive disease. Recent multi-institutional report highlighted excellent results obtained with 1-year LRF of $25 \%$, DMFS of $84 \%$ and OS of $65.2 \%$, respectively. These results were accompanied by low risk of acute Grade 3 toxicity (dysphagia, 9.1\%, mucositis, 9.9\%, esophagitis, 9.1\%, and dermatitis, 3.3\%), late Grades 3-4 dermatitis (8.7\%) and dysphagia (7.1\%) and Grade 5 bleeding in $2.9 \%$ patients.[93]

\section{Conclusion}

RT remains one of the cornerstones of treatment of HNC. This is so irrespective if it was given alone,[94] together with CHT[1,95] or in specific HNC patient populations.[2] Importantly, novel technological aspects of RT, such as IMRT, SRS, SBRT, or heavy particles significantly improved RT effectiveness on both $\mathrm{T}$ and $\mathrm{N}$ level. This was accompanied with decreased toxicity, making improved therapeutic benefit easily documented in contemporary clinical studies. Additional efforts should be made to further optimize these approaches in clinical studies within a framework of a more formal research setting.

Peer-review: Externally peer-reviewed.

Conflict of Interest: Authors declare no conflict of interest.

Financial Support: This work was partially funded by the grants from the Serbian Ministry of Education, Science and Technological Development III41007, ON174028.

\section{References}

1. Jeremic B, Dubinsky P, Filipovic N, Ozyigit G. Optimal administration frequency of cisplatin concurrently with radical radiotherapy in the definitive treatment of locally advanced, inoperable squamous cell cancer of the head and neck. Still obscured by clouds? Turk J Oncol 2019;34(2):133-6.

2. Jeremic B, Ozyigit G, Dubinsky P, Filipovic N. Importance of hpv positivity in squamous cell head and neck cancer. Turk J Oncol 2019;34(3):204-14.

3. Troost EG, Schinagl DA, Bussink J, Boermano C, van der Kogel AJ, Oyen WJ, et al. Innovations in radiotherapy planning of head and neck cancers: role of PET. J Nucl Med 2010;51(1):66-76.

4. Hendrickson K, Phillips M, Smith W, Peterson L, Krohn K, Rajendran J. Hypoxia imaging with [F-18] FMISO-PET in head and neck cancer: potential for guiding intensity modulated radiation therapy in overcoming hypoxia induced treatment resistance. Radiother Oncol 2011;101(3):369-75.

5. Chang JH, Wada M, Anderson NJ, Joon DL, Lee ST, Gong SJ, et al. Hypoxia-targeted radiotherapy dose painting for head and neck cancer using (18)FFMISO PET: a biological modeling study. Acta Oncol 2013;52(8):1723-9.

6. Lee N, Schoder H, Beattie B, Lanning R, Riaz N, McBride S, et al. Strategy of using intratreatment hypoxia imaging to selectively and safely guide radiation dose de-escalation concurrent with chemotherapy for locoregionally advanced human Papillomavirusrelated oropharyngeal carcinoma. Int J Radiat Oncol Biol Phys 2016;96(1):9-17.

7. Mortensen LS, Johansen J, Kallehauge J, Primdahl $\mathrm{H}$, Busk M, Lassen P, et al. FAZA PET/CT hypoxia imaging in patients with squamous cell carcinoma of the head and neck treated with radiotherapy: results from the DAHANCA 24 Trial. Radiother Oncol 2012;105(1):14-20.

8. Minagawa Y, Shizukuishi K, Koike I, Horiuchi C, Watanuki K, Hata M, et al. Assessment of tumor hypoxia by $62 \mathrm{Cu}-\mathrm{ATSM} \mathrm{PET} / \mathrm{CT}$ as a predictor of response in head and neck cancer: a pilot study. Ann Nucl Med 2011;25(5):339-45.

9. Grassi I, Nanni C, Cicoria G, Blasi C, Bunkheila F, Lopci E, et al. Usefulness of 64Cu-ATSM in head and neck cancer: a preliminary prospective study. Clin Nucl Med 2014;39(1):e59-63.

10. Geets X, Daisne JF, Gregoire V, Hamoir M, Lonneux M. Role of 11-C methionine positron emission tomography for the delineation of the tumor volume in pharyngo-laryngeal squamous cell carcinoma: comparison with FDG-PET and CT. Radiother Oncol 2004;71(3):267-73.

11. Hasebe M, Yoshikawa K, Ohashi S, Toubaru S, Kawaguchi K, Sato J, et al. A study on the prognostic evaluation of carbon ion radiotherapy for head and neck adenocarcinoma with C-11 methionine PET. Mol Imaging Biol 2010;12(5):554-62.

12. Buus S, Grau C, Munk OL, Bender D, Jensen K, Keiding S. 11C-Methionine PET, a novel method for measuring regional salivary gland function after radiotherapy of head and neck cancer. Radiother Oncol 2004;73(3):289-96.

13. Buus S, Grau C, Munk OL, Rodell A, Jensen K, Mouridsen $\mathrm{K}$, et al. Individual radiation response of parotid glands investigated by dynamic 11C-methionine PET. Radiother Oncol 2006;78(3):262-9.

14. Hoeben BA, Troost EG, Span PN, van Herpen CM, Bussink J, Oyen WJ, et al. 18F-FLT PET during radio- 
therapy or chemoradiotherapy in head and neck squamous cell carcinoma is an early predictor of outcome. J Nucl Med 2013;54(4):532-40.

15. Hoshikawa H, Yamamoto Y, Mori T, Kishino T, Fukumura T, Samukawa Y, et al. Predictive value of suvbased parameters derived from pre-treatment (18)FFLT PET/CT for short-term outcome with head and neck cancers. Ann Nucl Med 2014;28(10):1020-6.

16. Arens AI, Troost EG, Hoeben BA, Grootjans W, Lee JA, Grégoire V, et al. Semiautomatic methods for segmentation of the proliferative tumour volume on sequential FLT PET/CT images in head and neck carcinomas and their relation to clinical outcome. Eur J Nucl Med Mol Imaging 2014;41(5):915-24.

17. Kubiessa K, Purz S, Gawlitza M, Kühn A, Fuchs J, Steinhoff KG, et al. Initial clinical results of simultaneous 18F-FDG PET/MRI in comparison to 18F-FDG $\mathrm{PET} / \mathrm{CT}$ in patients with head and neck cancer. Eur J Nucl Med Mol Imaging 2014;41(4):639-48.

18. Samołyk-Kogaczewska N, Sierko E, Zuzda K, Gugnacki P, Szumowski P, Mojsak M, et al. PET/MRIguided GTV delineation during radiotherapy planning in patients with squamous cell carcinoma of the tongue. Strahlenther Onkol 2019;195(9):780-91.

19. Leibfarth S, Mönnich D, Welz S, Siegel C, Schwenzer N, Schmidt H, et al. A strategy for multimodal deformable image registration to integrate PET/MR into radiotherapy treatment planning. Acta Oncol 2013;52(7):1353-9.

20. Winter RM, Leibfarth S, Schmidt H, Zwirner K, Mönnich D, Welz S, et al. Assessment of image quality of a radiotherapy-specific hardware solution for PET/MRI in head and neck cancer patients. Radiother Oncol 2018;128(3):485-91.

21. Chao KS, Ozyigit G, Tran BN, Cengiz M, Dempsey JF, Low DA. Patterns of failure in patients receiving definitive and postoperative IMRT for head-and-neck cancer. Int J Radiat Oncol Biol Phys 2003;55(2):312-21.

22. Chao KS, Ozyigit G, Blanco AI, Thorstad WL, Deasy JO, Haughey BH, et al. Intensity-modulated radiation therapy for oropharyngeal carcinoma: impact of tumor volume. Int J Radiat Oncol Biol Phys 2004;59(1):43-50.

23. Mendenhall WM, Amdur RJ, Palta JR. Intensity-modulated radiotherapy in the standard management of head and neck cancer: promises and pitfalls. J Clin Oncol 2006;24(17):2618-23.

24. Vergeer MR, Doornaert PA, Rietveld DH, Leemans CR, Slotman BJ, Langendijk JA. Intensity-modulated radiotherapy reduces radiation-induced morbidity and improves health related quality of life: results of a nonrandomized prospective study using a standardized follow-up program. Int J Radiat Oncol Biol Phys
2009;74(1):1-8.

25. Marta GN, Silva V, de Andrade Carvalho H, de Arruda FF, Hanna SA, Gadia R, et al. Intensity-modulated radiation therapy for head and neck cancer: systematic review and meta-analysis. Radiother Oncol 2014;110(1):9-15.

26. Chao KS, Majhail N, Huang CJ, Simpson JR, Perez CA, Haughey $\mathrm{B}$, et al. Intensity-modulated radiation therapy reduces late salivary toxicity without compromising tumor control in patients with oropharyngeal carcinoma: a comparison with conventional techniques. Radiother Oncol 2001;61(2):275-80.

27. Beadle BM, Liao KP, Elting LS, Buchholz TA, Ang KK, Garden AS, et al. Improved survival using intensitymodulated radiation therapy in head and neck cancers: a SEER-medicare analysis. Cancer 2014;120(5):702-10.

28. Studer G, Zwahlen RA, Graetz KW, Davis BJ, Glanzmann C. IMRT in oral cavity cancer. Radiat Oncol 2007;2:16.

29. Zhang B, Mo Z, Du W, Wang Y, Liu L, Wei Y. Intensity-modulated radiation therapy versus $2 \mathrm{D}-\mathrm{RT}$ or $3 \mathrm{D}$ CRT for the treatment of nasopharyngeal carcinoma: a systematic review and meta-analysis. Oral Oncol 2015;51(11):1041-6.

30. Gupta T, Kannan S, Ghosh-Laskar S, Agarwal JP. Systematic review and meta-analyses of intensity-modulated radiation therapy versus conventional two-dimensional and/or or three-dimensional radiotherapy in curative-intent management of head and neck squamous cell carcinoma. PLoS One 2018;13(7):e0200137.

31. Ozyigit G, Chao KS. Clinical experience of headand-neck cancer IMRT with serial tomotherapy. Med Dosim 2002;27(2):91-8.

32. Jiang L, Zhang Y, Yang Z, Liang F, Wu J, Wang R. A comparison of clinical outcomes between simultaneous integrated boost (SIB) versus sequential boost (SEQ) intensity modulated radiation therapy (IMRT) for head and neck cancer: a meta-analysis. Medicine (Baltimore) 2019;34:e16942.

33. Ho KF, Fowler JF, Sykes AJ, Yap BK, Lee LW, Slevin NJ. IMRT dose fractionation for head and neck cancer: variation in current approaches will make standardization difficult. Acta Oncol 2009; 48(3):431-9.

34. Spiotto MT, Weichselbaum RR. comparison of $3 \mathrm{D}$ conformal radiotherapy and intensity modulated radiotherapy with or without simultaneous integrated boost during concurrent chemoradiation for locally advanced head and neck cancers. PLoS One 2014;9:e94456.

35. Vlacich G, Stavas MJ, Pendyala P, Chen SC, Shyr Y, Cmelak AJ. A comparative analysis between sequential boost and integrated boost intensity-modulated 
radiation therapy with concurrent chemotherapy for locally-advanced head and neck cancer. Radiat Oncol 2017;12(1):13.

36. Vanetti E, Clivio A, Nicolini G, Fogliata A, GhoshLaskar S, Agarwal JP, et al. Volumetric modulated arc radiotherapy for carcinomas of the oro-pharynx, hypo-pharynx and larynx: a treatment planning comparison with fixed field IMRT. Radiother Oncol 2009;92(1):111-7.

37. Matsuzak MM, Yan D, Grills I, Martinez A. Clinical applications of volumetric modulated arc therapy. Int J Radiat Oncol Biol Phys 2010;77(2):608-16.

38. Bertelsen A, Hansen CR, Johansen J, Brink C. Single arc volumetric modulated arc therapy of head and neck cancer. Radiother Oncol 2010;95(2):142-8.

39. Clemente S, Wu B, Sanguineti G, Fusco V, Ricchetti F, Wong J, et al. Smart arc-based volumetric modulated arc therapy for oropharyngeal cancer: a dosimetric comparison with both intensity-modulated radiation therapy and helical tomotherapy. Int J Radiat Oncol Biol Phys 2011;80(4):1248-55.

40. Van Gestel D, van Vliet-Vroegindeweij C, Van den Heuvel F, Crijns W, Coelmont A, De Ost B, et al. RapidArc, SmartArc and TomoHD compared with classical step and shoot and sliding window intensity modulated radiotherapy in an oropharyngeal cancer treatment plan comparison. Radiat Oncol 2013;8:37.

41. Chang SD, Tate DJ, Goffinet DR, Martin DP, Adler JR Jr. Treatment of nasopharyngeal carcinoma: stereotactic radiosurgery boost following fractionated radiotherapy. Stereotact Funct Neurosurg 1999;73(1-4):64-7.

42. Ahn YC, Lee CK, Kin DY, Huh SJ, Yeo IH, Lim DH, et al. Fractionated stereotactic radiation therapy for extracranial head and neck tumors. Int J Radiat Oncol Biol Phys 2000;48(2):501-5.

43. Siddiqui F, Patel M, Khan M, McLean S, Dragovic J, Jin JY, et al. Stereotactic body radiation therapy for primary, recurrent, and metastatic tumors in the head-and-neck region. Int J Radiat Oncol Biol Phys 2009;74(4):1047-53.

44. Kodani N, Yamazaki H, Tsubokura T, Shiomi H, Kobayashi K, Nishimura T, et al. Stereotactic body radiation therapy for head and neck tumor: disease control and morbidity outcomes. J Radiat Res 2011;52(1):24-31.

45. Baker S, Verduijn G, Petit S, Nuyttens JJ, Sewnaik A, van der Lugt $A$, et al. Locoregional failures and their relation to radiation fields following stereotactic body radiotherapy boost for oropharyngeal squamous cell carcinoma. Head Neck 2019;41(6):1622-31.

46. Díaz-Martínez JA, Esquenazi Y, Martir M, Citardi MJ, Karni RJ, Blanco AI. Planned gamma knife boost after chemoradiotherapy for selected sinonasal and nasopharyngeal cancers. World Neurosurg 2018;119:e467-74.

47. Ozyigit G, Cengiz M, Hurmuz P, Yazici G, Gultekin $\mathrm{M}$, Akyol F, et al. Robotic stereotactic radiosurgery in patients with nasal cavity and paranasal sinus tumors. Technol Cancer Res Treat 2014;13(5):409-13.

48. Ho JC, Phan J. Reirradiation of head and neck cancer using modern highly conformal techniques. Head Neck 2018;40(9):2078-93.

49. Ozyigit G, Cengiz M, Yazici G, Yildiz F, Gurkaynak M, Zorlu F, et al. A retrospective comparison of robotic stereotactic body radiotherapy and three-dimensional conformal radiotherapy for the reirradiation of locally recurrent nasopharyngeal carcinoma. Int J Radiat Oncol Biol Phys 2011;81(4):e263-8.

50. Alterio D, Marvaso G, Ferrari A, Volpe S, Orecchia R, Jereczek-Fossa BA. Modern radiotherapy for head and neck cancer. Semin Oncol 2019;46(3):233-45.

51. Janot F, de Raucourt D, Benhamou E, Ferron C, Dolivet G, Bensadoun RJ, et al. Randomized trial of postoperative reirradiation combined with chemotherapy after salvage surgery compared with salvage surgery alone in head and neck carcinoma. J Clin Oncol 2008;26(34):5518-23.

52. Cengiz M, Özyiğit G, Yazici G, Doğan A, Yildiz F, Zorlu F, et al. Salvage reirradiaton with stereotactic body radiotherapy for locally recurrent head-and-neck tumors. Int J Radiat Oncol Biol Phys 2011;81(1):104-9.

53. Yazici G, Sanlı TY, Cengiz M, Yuce D, Gultekin M, Hurmuz P, et al. A simple strategy to decrease fatal carotid blowout syndrome after stereotactic body reirradiaton for recurrent head and neck cancers. Radiat Oncol 2013;8:242.

54. Oda K, Mori Y, Kobayashi T, Kida Y, Yokoi H, Shibamoto $\mathrm{Y}$, et al. Stereotactic radiosurgery as a salvage treatment for recurrent epipharyngeal carcinoma. stereotact funct neurosurg 2006;84(2-3):103-8.

55. Kohler RE, Sheets NC, Wheeler SB, Nutting C, Hall E, Chera BS. Two-year and lifetime cost-effectiveness of intensity modulated radiation therapy versus 3 -dimensional conformal radiation therapy for head-and-neck cancer. Int J Radiat Oncol Biol Phys 2013;87(4):683-9.

56. Marta GN, Weltman E, Ferrigno R. Intensity-modulated radiation therapy (IMRT) versus 3-dimensional conformal radiation therapy (3D-CRT) for head and neck cancer: cost-effectiveness analysis. Rev Assoc Bras 2018;64(4):318-23.

57. Ikawa H, Koto M, Demizu Y, Saitoh JI, Suefuji H, Okimoto $\mathrm{T}$, et al. Multicenter study of carbon-ion radiation therapy for nonsquamous cell carcinomas of the oral cavity. Cancer Med 2019;8(12):5482-91. 
58. Shirai K, Koto M, Demizu Y, Suefuji H, Ohno T, Tsuji $\mathrm{H}$, et al. Multi-institutional retrospective study of mucoepidermoid carcinoma treated with carbon-ion radiotherapy. Cancer Sci 2017;108(7):1447-51.

59. Held T, Windisch P, Akbaba S, Lang K, El Shafie R, Bernhardt $\mathrm{D}$, et al. Carbon ion reirradiation for recurrent head and neck cancer: a single-institutional experience. Int J Radiat Oncol Biol Phys 2019;105(4):803-11.

60. Akbaba S, Held T, Lang K, Forster T, Federspil P, Herfarth $\mathrm{K}$, et al. Bimodal radiotherapy with active rasterscanning carbon ion radiotherapy and intensitymodulated radiotherapy in high-risk nasopharyngeal carcinoma results in excellent local control. Cancers (Basel) 2019;11(3):379.

61. Akbaba S, Ahmed D, Lang K, Held T, Mattke M, Hoerner-Rieber J, et al. Results of a combination treatment with intensity modulated radiotherapy and active raster-scanning carbon ion boost for adenoid cystic carcinoma of the minor salivary glands of the nasopharynx. Oral Oncol 2019;91(1):39-46.

62. Adeberg S, Akbaba S, Lang K, Held T, Verma V, Nikoghosyan A, et al. The phase 1/2 ACCEPT Trial: concurrent cetuximab and intensity-modulated radiotherapy with carbon ion boost for adenoid cystic carcinoma of the head and neck. Int J Radiat Oncol Biol Phys 2020;106(1):167-73.

63. Jensen AD, Nikoghosyan AV, Lossner K, Haberer T, Jäkel O, Münter MW, et al. COSMIC: a regimen of intensity modulated radiation therapy plus dose-escalated, raster-scanned carbon ion boost for malignant salivary gland tumors: results of the prospective phase 2 trial. Int J Radiat Oncol Biol Phys 2015;93(1):37-46.

64. Buchholz TA, Laramore GE, Griffin BW, Koh WJ, Griffin TW. The role of fast neutron radiation therapy in the management of advanced salivary gland malignant neoplasms. Cancer 1992;69(11):2779-88.

65. Huber PE, Debus J, Latz D, Zierhut D, Bischof M, Wannenmacher $\mathrm{M}$, et al. Radiotherapy for advanced adenoid cystic carcinoma: neutrons, photons or mixed beam? Radiother Oncol 2001;59(2):161-7.

66. Stannard C, Vernimmen F, Carrara H, Jones D, Fredericks S, Hille J, et al. Malignant salivary gland tumours: can fast neutron therapy results point the way to carbon ion therapy? Radiother Oncol 2013;109(2):262-8.

67. Maor MH, Errington RD, Caplan RJ, Griffin TW, Laramore GE, Parker RG, et al. Fast-neutron therapy in advanced head and neck cancer: a collaborative international randomized trial. Int J Radiat Oncol Biol Phys 1995;32(3):599-604.

68. van de Water TA, Bijl HP, Schilstra C, Pijls-Johannesma $M$, Langendijk JA. The potential benefit of radiotherapy with protons in head and neck cancer with respect to normal tissue sparing: a systematic review of literature. oncologist 2011;16(3):366-77.

69. Lomax AJ, Goitein M, Adams J. Intensity modulation in radiotherapy: photons versus protons in the paranasal sinus. Radiother Oncol 2003;66(1):11-8.

70. Widesott L, Pierelli A, Fiorino C, Delloca I, Broggi S, Cattaneo GM, et al. Intensity-modulated proton therapy versus helical tomotherapy in nasopharynx cancer: planning comparison and NTCP evaluation. Int J Radiat Oncol Biol Phys 2008;72(2):589-96.

71.van der Laan HP, van de Water TA, van Herpt HE, Christianen ME, Bijl HP, Korevaar EW, et al. The potential of intensity-modulated proton radiotherapy to reduce swallowing dysfunction in the treatment of head and neck cancer: a planning comparative study. Acta Oncol 2013;52(3):561-9.

72. Kandula S, Zhu X, Garden AS, Gillin M, Rosenthal DI, Ang KK, et al. Spot-scanning beam proton therapy vs. Intensity-modulated radiation therapy for ipsilateral head and neck malignancies: a treatment planning comparison. Med Dosim 2013;38(4):390-4.

73. Lomax AJ. Intensity modulated proton therapy and its sensitivity to treatment uncertainties 1 : the potential effects of calculational uncertainties. Phys Med Biol 2008;53(4):1027-42.

74. Quan EM, Liu W, Wu R, Li Y, Frank SJ, Zhang X, et al. Preliminary evaluation of multifield and single field optimization for the treatment planning of spot-scanning proton therapy of head and neck cancer. Med Phys 2013;40(8):081709.

75. Kraan AC, van de Water S, Teguh DN, Al-Mamgani A, Madden T, Kooy HM, et al. Dose uncertainties in IMPT for oropharyngeal cancer in the presence of anatomical, range, and setup errors. Int J Radiat Oncol Biol Phys 2013;87(5):888-96.

76. Apinorasethkul O, Kirk M, Teo K, Swisher-McClure S, Lukens JN, Lin A. Pencil beam scanning proton therapy vs rotational arc radiation therapy: a treatment planning comparison for postoperative oropharyngeal cancer. Med Dosim 2017;42(1):7-11.

77. Fukumitsu N, Okumura T, Mizumoto M, Oshiro Y, Hashimoto T, Kanemoto A, et al. Outcome of T4 (International Union Against Cancer Staging System, 7th edition) or recurrent nasal cavity and paranasal sinus carcinoma treated with proton beam. Int J Radiat Oncol Biol Phys 2012;83(2):704-11.

78. Rutz HP, Weber DC, Goitein G, Ares C, Bolsi A, Lo$\max \mathrm{AJ}$, et al. Postoperative spot-scanning proton radiation therapy for chordoma and chondrosarcoma in children and adolescents: initial experience at Paul Scherrer Institute. Int J Radiat Oncol Biol Phys 2008;71(1):220-25. 
79. Ares C, Hug EB, Lomax AJ, Bolsi A, Timmermann B, Rutz HP, et al. Effectiveness and safety of spot scanning proton radiation therapy for chordomas and chondrosarcomas of the skull base: first long ᄀ-term report. Int J Radiat Oncol Biol Phys 2009;75(4):1111-18.

80. Chan A, Adams JA, Weyman E. A Phase II trial of proton radiation therapy with chemotherapy for nasopharyngeal carcinoma. Int J Radiat Oncol Biol Phys 2012;84:S151-52.

81. Holliday EB, Garden AS, Rosenthal DI, Fuller CD, Morrison WH, Gunn GB, et al. Proton therapy reduces treatment $\neg$ related toxicities for patients with nasopharyngeal cancer: a case $\neg$ match control study of intensity $\neg$ modulated proton therapy and intensity $\neg$ modulated photon therapy. Int J Particle Ther 2015;2(1):19-28.

82. McDonald MW, Liu Y, Moore MG, Johnstone PA. acute toxicity in comprehensive head and neck radiation for nasopharynx and paranasal sinus cancers: cohort comparison of 3D conformal proton therapy and intensity modulated radiation therapy. Radiat Oncol 2016;11:32.

83. Truong MT, Kamat UR, Liebsch NJ, Curry WT, Lin DT, Barker FG 2nd, et al. Proton radiation therapy for primary sphenoid sinus malignancies: treatment outcome and prognostic factors. Head Neck 2009;31(10):1297-308.

84. Russo AL, Adams JA, Weyman EA, Busse PM, Goldberg SI, Varvares M, et al. Long-term outcomes after proton beam therapy for sinonasal squamous cell carcinoma. Int J Radiat Oncol Biol Phys 2016;95(1):36876.

85. Dagan R, Bryant C, Li Z, Yeung D, Justice J, Dzieglewiski $\mathrm{P}$, et al. Outcomes of sinonasal cancer treated with proton therapy. Int J Radiat Oncol Biol Phys 2016;95(1):377-85.

86. Nakamura T, Azami Y, Ono T, Yamaguchi H, Hayashi Y, Suzuki M, et al. Preliminary results of proton beam therapy combined with weekly cisplatin intraarterial infusion via a superficial temporal artery for treatment of maxillary sinus carcinoma. Jpn J Clin Oncol 2016;46(1):46-50.

87. El-Sawy T, Frank SJ, Hanna E, Sniegowski M, Lai SY,
Nasser QJ, et al. Multidisciplinary management of lacrimal sac/nasolacrimal duct carcinomas. Ophthal Plast Reconstr Surg 2013;29(6):454-7.

88. Holliday EB, Esmaeli B, Pinckard J, Garden AS, Rosenthal DI, Morrison WH, et al. A Multidisciplinary orbitsparing treatment approach that includes proton therapy for epithelial tumors of the orbit and ocular adnexa. Int J Radiat Oncol Biol Phys 2016;95(1):344-52.

89. Slater JD, Yonemoto LT, Mantik DW, Bush DA, Preston W, Grove RI, et al. Proton radiation for treatment of cancer of the oropharynx: early experience at Loma Linda University medical center using a concomitant boost technique. Int J Radiat Oncol Biol Phys 2005;62(2):494-500.

90. Hutcheson K, Lewin JS, Garden AS. Early experience with IMPT for the treatment of oropharyngeal tumors: acute toxicities and swallowing-related outcomes. Int J Radiat Oncol Biol Phys 2013;87:S604.

91. Blanchard P, Garden AS, Gunn GB, Rosenthal DI, Morrison WH, Hernandez M, et al. Intensity $\neg$ modulated proton beam therapy (IMPT) versus intensity $\neg$ modulated photon therapy (IMRT) for patients with oropharynx cancer a case matched analysis. Radiother Oncol 2016;120(1):48-55.

92. Gunn GB, Blanchard P, Garden AS, Zhu XR, Fuller $\mathrm{CD}$, Mohamed AS, et al. Clinical outcomes and patterns of disease recurrence after intensity modulated proton therapy for oropharyngeal squamous carcinoma. Int J Radiat Oncol Biol Phys 2016;95(1):360-7.

93. Romesser PB, Cahlon O, Scher ED, Hug EB, Sine K, DeSelm C, et al. Proton beam reirradiation for recurrent head and neck cancer: multi-institutional report on feasibility and early outcomes. Int J Radiat Oncol Biol Phys 2016;95(1):386-95.

94. Jeremic B, Ozyigit G, Jeremic M, Dubinsky P. Impacts of radiotherapy fractionation on outcome in squamous cell head and neck cancer (SQC HNC). Turk J Oncol 2020;35(3):356-62.

95. Jeremic B, Dubinsky P, Jeremic M, Kiladze I, Ozyigit G. Optimal dose of cisplatin (CDDP) given concurrently with radiotherapy (RT) in locally advanced squamous cell head and neck cancer (SQC HNC). Turk J Oncol 2020;35(2):237-41. 